\title{
Effect of whole-body vibration therapy on lower extremity function in subacute stroke patients
}

\author{
Jin Wan Kim, Jong Hwa Lee* \\ Department of Physical Medicine and Rehabilitation, Dong-A University College of Medicine, Busan, Korea
}

This study aimed to investigate the effect of whole-body vibration therapy on lower extremity function in subacute stroke patients. Subacute stroke patients who were able to undergo gait training were randomly divided into a vibration therapy group and a control group. All patients attended 20-min training sessions twice daily, 5 times a week for 2 weeks. Each session included 45 squats. The vibration group trained on a vibration platform and the control group trained on the ground. The degree of maximal isokinetic voluntary contraction torque was evaluated, and manual muscle tests of hip and knee flexion and extension were performed. The Berg Balance Scale, 10-m walk test, Timed Up and Go Test, and Functional Ambulation Category were used. A total of 38 patients, 20 in the vibration group and 18 in the control group, were included in the analysis. After the 2-week therapy, the vibration group showed significant improvements in lower extremity strength, balance, and gait performance. The vibration group showed significantly better performance on the Berg Balance Scale, 10-m walk test, and Functional Ambulation Category than the control group. There were no significant differences in maximal isokinetic voluntary contraction torque or manual muscle tests between the groups. Our results suggest that additional training with whole-body vibration may effectively improve the balance and gait performance of subacute stroke patients. Further studies on large populations are required to determine the therapy's clinical efficacy.

Keywords: Whole-body vibration therapy, Subacute stroke, Lower extremity function

\section{INTRODUCTION}

Stroke is a serious medical condition with various complications caused by brain damage, including motor weakness, sensory deficit, balance and/or proprioception impairment, cognitive impairment, language problems, and swallowing difficulties. Gait disturbance and impaired functional mobility are common problems in stroke patients. As independent transfer is vital to performing activities of daily living, efforts to improve gait function are a crucial part of rehabilitation. Many types of treatment have been developed to that end, including therapeutic exercise, electrical stimulation, transcranial magnetic stimulation, and robotics-assisted gait training.

Whole-body vibration (WBV) therapy has recently been investigated as a rehabilitation method for athletes and patients. It has been increasingly used in clinical practice for improving neu- ro-motor performance in various patient populations. Mechanical vibration at different frequencies and intensities has been widely used to improve muscle function and performance (Martinez et al., 2013; Torvinen et al., 2002). The vibration signals activate the muscle spindles, inducing reflexive activation of motor units (Pang et al., 2006). Thus, WBV training may reduce disability by improving balance, gait performance, and mobility in stroke patients (Yang et al., 2015). However, although several studies have demonstrated the effects of WBV therapy on the functional recovery of the lower extremities, its benefit for subacute stroke patients has not been well established. Most rehabilitation therapies are known to be more effective when applied at an early stage. Thus, we hypothesized that early WBV therapy would be more effective in improving balance and gait performance. Therefore, the aim of this study was to investigate the effect of WBV therapy on lower extremity function in subacute stroke patients.
${ }^{*}$ Corresponding author: Jong Hwa Lee (D) https://orcid.org/0000-0003-2489-358X Department of Physical Medicine and Rehabilitation, Dong-A University College of Medicine, 26 Daesingongwon-ro, Seo-gu, Busan 49201, Korea Email:jhlee08@dau.ac.kr

Received: April 20, 2021 / Accepted: May 15, 2021
This is an Open Access article distributed under the terms of the Creative Commons Attribution Non-Commercial License (https://creativecommons.org/licenses/by-nc/4.0/) which permits unrestricted non-commercial use, distribution, and reproduction in any medium, provided the original work is properly cited. 


\section{MATERIALS AND METHODS}

\section{Participants}

The study enrolled patients who referred to our medical center with a stroke. The participants were first-onset subacute stroke patients within 2 months of the incident and had motor weakness with walking disability. Patients were eligible if their scores in the Korean version of the Mini-Mental State Examination were 10 or above and their motor power of hip and knee extension was 3 or above on the Medical Research Council scale to ensure that they could undergo standing and gait training. Patients who had a cerebrovascular incident history or non-stroke-related sensory or motor impairments, used medications that could interfere with postural control, did not cooperate in assessment and therapy, or had contraindications for WBV, such as recent fractures, malignancies, or a cardiac pacemaker, were excluded from the study.

Forty patients met the inclusion criteria. These patients were randomly divided into a WBV therapy group and a control group. Two participants could not be followed up because of health problems (Fig. 1). Therefore, 38 patients, 20 in the WBV group and 18 in the control group, completed the therapy and were included in the analysis. G Power 3.1 was used to calculate the required sample size. Based on a power of $80 \%$ and a twotailed alpha of 0.05 , we calculated that the sample size required per group was 18. Assuming a $10 \%$ loss to follow-up, we estimated that the final sample size required was 20 per group-that is, a total of 40 patients.

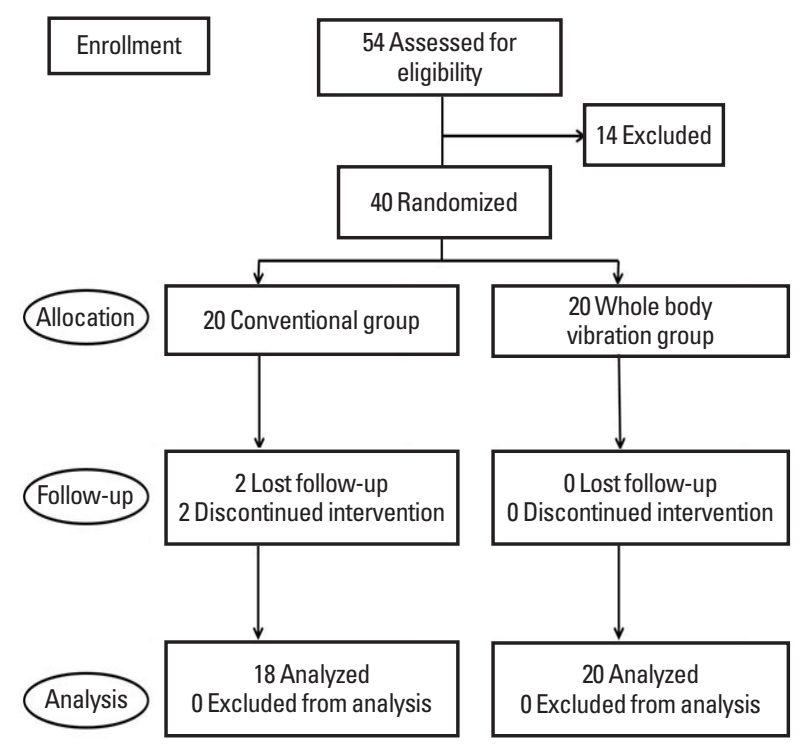

Fig. 1. Study flow diagram.

\section{Methods}

All participants attended 20-min conventional therapy sessions twice daily, 5 times a week for 2 weeks. The therapy included standing balance and gait training provided by a physical therapist. In each session, the patients in both groups additionally performed 45 squats with the therapist's assistance as appropriate. The patients in the WBV therapy group trained on a vibration platform (Sonix SW-VM10; Sonic World, Wonju, Korea), while those in the control group trained on the ground.

According to a systematic review and meta-analysis, various frequencies have been tested in WBV therapy to date, ranging from 5 to $40 \mathrm{~Hz}$ (Yang et al., 2015). However, there are currently no guidelines regarding the appropriate frequency and amplitude for WBV therapy in stroke patients. According to the instrument's guide, the recommended frequency for lower extremity training is $16 \mathrm{~Hz}$. Since it was within the range of previous studies, we selected that frequency. The intensity of the stimulus provided by the instrument is adjustable to a range from 0 to 99 . After several trials, we set the intensity to 40 , a moderate intensity for the patients.

Before the intervention, the participants' age, gender, location and type of lesions, location of paralysis, time from stroke onset, and Mini-Mental State Examination (Korean version) scores were recorded. The degree of maximal isokinetic voluntary contraction torque was evaluated, and manual muscle tests were performed to evaluate lower extremity strength. The Berg Balance Scale was used to evaluate balance. The 10-m walking test, Timed Up and Go Test, and Functional Ambulation Category were used to evaluate gait performance. These evaluations were performed at baseline and immediately after the 2-week therapy.

Maximal isokinetic voluntary contraction torque was measured on hip and knee flexion and extension on the affected side using an isokinetic multijoint test system (Biodex System 4, Biodex Medical Systems, Shirley, NY, USA). The highest values were derived from three measurements for each. Manual muscle tests were performed on hip and knee flexion and extension on the affected side according to the traditional method (G0-G5). The Berg Balance Scale tasks were performed according to the tester's instructions. Each item was rated on a scale from 0 to 4 points, with a maximum total of 56 points. Higher scores indicate higher levels of function. In the 10-m walk test, each patient was asked to walk a distance of $14 \mathrm{~m}$, and the time it took to cover $10 \mathrm{~m}$ was recorded. The Timed Up and Go Test measured how long it took the patient to get up from a chair with an armrest, walk $3 \mathrm{~m}$, change direction, and return to the chair. The Functional Ambu- 
lation Category was rated from 0 to 5 , depending on the degree to which the patient required assistance to walk. 0 represents inability to walk or requiring assistance from two or more persons, and 5 represents ability to walk independently anywhere.

\section{Statistical analysis}

Statistical analysis was performed using IBM SPSS ver. 18.0 (IBM Co., Armonk, NY, USA). The Mann-Whitney U-test was used for comparisons between the two groups. The Wilcoxon signed-rank test was used to evaluate differences in each patient's maximal isokinetic voluntary contraction torque, manual muscle tests, Berg Balance Scale, 10-m walk test, Timed Up and Go Test, and Functional Ambulation Category before and after treatment. The level of statistical significance was set to $P<0.05$.

\section{Ethics}

The experimental procedures in this study complied with the 1983 revision of the 1975 Declaration of Helsinki. Informed consent was obtained from all participants after full explanations of the study's purpose and methodology according to the ethical guidelines of our hospital. The study was approved by the hospital's ethics committee (DAUHIRB-20-032).

\section{RESULTS}

Table 1 shows the baseline characteristics of the participants. There were no significant differences in age, gender, affected side, type of stroke, time from stroke onset, and baseline Mini-Mental State Examination, manual muscle tests, maximal isokinetic voluntary contraction torque, Berg Balance Scale, 10-m walk test, Timed Up and Go Test, and Functional Ambulation Category between the two groups.

After 2 weeks of therapy, the patients in both groups showed significant improvement in the manual muscle test of hip and knee flexion and extension $(P<0.05)$. In the maximal isokinetic voluntary contraction torque of hip and knee flexion and knee extension, both groups also showed significant improvement $(P<$ 0.05) (Table 2).

The patients in both groups also significantly improved their Berg Balance Scale and Functional Ambulation Category scores $(P<0.05)$. The WBV group also showed statistically significant improvement in the 10 -m walk test $(P<0.05)$. Neither group showed significant improvement in the Timed Up and Go Test $(P>0.05)$ (Table 2).

The WBV group showed significantly greater improvement in
Table 1. Baseline characteristics of the two groups

\begin{tabular}{lccc}
\hline Characteristic & $V G(n=20)$ & $C G(n=18)$ & $P$-value \\
\hline Age (yr) & $57.20 \pm 11.00$ & $55.70 \pm 10.40$ & 0.173 \\
Sex, male:female & $8: 12$ & $7: 11$ & 0.850 \\
Lesion & & & \\
Right:left & $11: 9$ & $7: 11$ & 0.465 \\
Ischemic:hemorrhagic & $15: 5$ & $10: 8$ & 0.206 \\
Supratentorial: & $16: 4$ & $15: 3$ & 0.850 \\
$\quad$ infratentorial & & & \\
Day from stroke onset & $31.60 \pm 15.18$ & $28.00 \pm 8.72$ & 0.134 \\
K-MMSE & $18.60 \pm 6.70$ & $20.33 \pm 2.08$ & 0.356 \\
MMT & & & \\
HF & $3.10 \pm 0.55$ & $3.33 \pm 0.58$ & 0.395 \\
HE & $3.20 \pm 0.51$ & $3.25 \pm 0.30$ & 0.255 \\
KF & $3.21 \pm 0.25$ & $3.02 \pm 0.45$ & 0.455 \\
KE & $3.60 \pm 0.55$ & $3.30 \pm 0.23$ & 0.495 \\
MIT & & & \\
HF (N.m) & $29.40 \pm 5.72$ & $31.67 \pm 7.51$ & 0.072 \\
HE (N.m) & $22.60 \pm 5.94$ & $23.67 \pm 4.73$ & 0.174 \\
KF (N.m) & $24.60 \pm 6.11$ & $22.33 \pm 6.81$ & 0.153 \\
KE (N.m) & $33.40 \pm 5.13$ & $31.33 \pm 8.50$ & 0.230 \\
BBS & $35.40 \pm 8.14$ & $36.00 \pm 12.17$ & 0.272 \\
1OMWT (sec) & $24.00 \pm 5.79$ & $22.45 \pm 7.55$ & 0.151 \\
TUGT (sec) & $22.00 \pm 6.12$ & $19.67 \pm 6.43$ & 0.252 \\
FAC & $3.20 \pm 0.45$ & $3.37 \pm 0.58$ & 0.271 \\
\hline Val & & &
\end{tabular}

Values are numbers or mean \pm standard deviation or number.

VG, vibration group; CG, control group; K-MMSE, Korean version of Mini-Mental State Examination; MMT, manual muscle test, affected side; HF, hip flexor; HE, hip extensor; KF, knee flexor; KE, knee extensor; MIT, maximal isokinetic voluntary contraction torque, affected side; BBS, Berg Balance Scale; 10MWT, 10-m walking test; TUGT, Timed Up and Go Test; FAC, Functional Ambulation Category.

the Berg Balance Scale, 10-m walk test, and Functional Ambulation Category than the control group $(P<0.05)$. Conversely, there were no statistically significant differences in maximal isokinetic voluntary contraction torque, manual muscle tests, or the Timed Up and Go Test between the two groups $(P>0.05)$ (Table 3).

\section{DISCUSSION}

In this study, we compared the effects of WBV therapy with those of conventional rehabilitation therapy on the lower extremity function of subacute stroke patients. After the two-week therapy, the WBV group showed significant improvement in lower extremity strength, balance, and gait performance, as measured by manual muscle tests, maximal isokinetic voluntary contraction torque, and the Berg Balance Scale, 10-m walk test, and Functional Ambulation Category. The control group also showed statistically significant improvement in the manual muscle tests, 
Table 2. Changes of measurements by the therapy and clinical correlation between the two groups

\begin{tabular}{|c|c|c|c|c|c|c|}
\hline \multirow{2}{*}{ Variable } & \multicolumn{2}{|c|}{$V G(n=20)$} & \multirow{2}{*}{$P$-value } & \multicolumn{2}{|c|}{ CG $(n=18)$} & \multirow{2}{*}{$P$-value } \\
\hline & Pre & Post & & Pre & Post & \\
\hline \multicolumn{7}{|l|}{ MMT } \\
\hline $\mathrm{HF}$ & $3.10 \pm 0.55$ & $3.60 \pm 0.25$ & $0.005^{*}$ & $3.33 \pm 0.58$ & $3.67 \pm 0.38$ & $0.015^{*}$ \\
\hline HE & $3.20 \pm 0.51$ & $3.70 \pm 0.46$ & $0.005^{*}$ & $3.25 \pm 0.30$ & $3.60 \pm 0.52$ & $0.020^{*}$ \\
\hline KF & $3.21 \pm 0.25$ & $3.60 \pm 0.63$ & $0.025^{*}$ & $3.02 \pm 0.45$ & $3.35 \pm 0.51$ & $0.007^{*}$ \\
\hline KE & $3.60 \pm 0.55$ & $4.10 \pm 0.55$ & $0.001^{*}$ & $3.30 \pm 0.23$ & $3.65 \pm 0.23$ & $0.010^{*}$ \\
\hline \multicolumn{7}{|l|}{ MIT } \\
\hline$H F(N \cdot m)$ & $29.40 \pm 5.72$ & $35.60 \pm 4.93$ & $0.032^{*}$ & $31.67 \pm 7.51$ & $35.90 \pm 5.54$ & $0.017^{*}$ \\
\hline $\mathrm{HE}(\mathrm{N} \cdot \mathrm{m})$ & $22.60 \pm 5.94$ & $29.20 \pm 5.50$ & $0.003^{*}$ & $23.67 \pm 4.73$ & $27.67 \pm 6.43$ & $0.047^{*}$ \\
\hline $\mathrm{KF}(\mathrm{N} \cdot \mathrm{m})$ & $24.60 \pm 6.11$ & $30.60 \pm 4.22$ & $0.030^{*}$ & $22.33 \pm 6.81$ & $27.33 \pm 4.51$ & $0.003^{*}$ \\
\hline $\mathrm{KE}(\mathrm{N} \cdot \mathrm{m})$ & $33.40 \pm 5.13$ & $39.80 \pm 4.38$ & $0.005^{*}$ & $31.33 \pm 8.50$ & $36.33 \pm 6.21$ & $0.002^{*}$ \\
\hline BBS & $35.40 \pm 8.14$ & $46.80 \pm 6.14$ & $0.002^{*}$ & $36.00 \pm 12.17$ & $40.00 \pm 15.62$ & $0.027^{*}$ \\
\hline 10MWT (sec) & $24.00 \pm 5.79$ & $18.00 \pm 3.54$ & $0.032^{*}$ & $22.45 \pm 7.55$ & $19.67 \pm 8.74$ & 0.109 \\
\hline TUGT (sec) & $22.00 \pm 6.12$ & $20.20 \pm 3.63$ & 0.068 & $19.67 \pm 6.43$ & $18.33 \pm 6.81$ & 0.102 \\
\hline FAC & $3.20 \pm 0.45$ & $4.30 \pm 0.50$ & $0.006^{*}$ & $3.37 \pm 0.58$ & $3.70 \pm 1.20$ & $0.035^{*}$ \\
\hline
\end{tabular}

Values are presented as mean \pm standard deviation.

VG, vibration group; CG, control group; MMT, manual muscle test, affected side; HF, hip flexor; HE, hip extensor; KF, knee flexor; KE, knee extensor; MIT, maximal isokinetic voluntary contraction torque, affected side; BBS, Berg Balance Scale; 10MWT, 10-meter walking test; TUGT, Timed Up and Go Test; FAC, Functional Ambulation Category.

${ }^{*} P<0.05$ by Wilcoxon signed-rank test.

Table 3. Comparison of the therapeutic effect between the two groups

\begin{tabular}{lccc}
\hline Variable & $\mathrm{VG}(\mathrm{n}=20)$ & $\mathrm{CG}(\mathrm{n}=18)$ & $P$-value \\
\hline $\begin{array}{l}\Delta \mathrm{MMT} \\
\mathrm{HF}\end{array}$ & $0.50 \pm 0.06$ & $0.34 \pm 0.30$ & 0.089 \\
$\mathrm{HE}$ & $0.50 \pm 0.12$ & $0.35 \pm 0.58$ & 0.135 \\
$\mathrm{KF}$ & $0.39 \pm 0.23$ & $0.33 \pm 0.18$ & 0.754 \\
$\mathrm{KE}$ & $0.51 \pm 0.15$ & $0.35 \pm 0.20$ & 0.059 \\
$\Delta \mathrm{MIT}$ & & & \\
$\mathrm{HF}(\mathrm{N} \cdot \mathrm{m})$ & $6.20 \pm 2.49$ & $4.33 \pm 2.31$ & 0.079 \\
$\mathrm{HE}(\mathrm{N} \cdot \mathrm{m})$ & $6.60 \pm 2.07$ & $4.00 \pm 1.73$ & 0.064 \\
$\mathrm{KF}(\mathrm{N} \cdot \mathrm{m})$ & $6.00 \pm 2.24$ & $5.00 \pm 1.52$ & 0.535 \\
$\mathrm{KE}(\mathrm{N} \cdot \mathrm{m})$ & $6.20 \pm 0.89$ & $5.00 \pm 1.73$ & 0.530 \\
$\Delta \mathrm{BBS}$ & $11.40 \pm 2.88$ & $4.00 \pm 3.46$ & $0.012^{*}$ \\
$\Delta 10 \mathrm{MWT}(\mathrm{sec})$ & $-6.00 \pm 2.35$ & $-3.33 \pm 1.53$ & $0.037^{*}$ \\
$\Delta \mathrm{TUGT}(\mathrm{sec})$ & $-1.80 \pm 1.59$ & $-1.33 \pm 0.58$ & 0.539 \\
$\Delta \mathrm{FAC}$ & $1.10 \pm 0.45$ & $0.33 \pm 0.48$ & $0.043^{*}$ \\
\hline
\end{tabular}

Values are presented as mean \pm standard deviation.

$\mathrm{VG}$, vibration group; $\mathrm{CG}$, control group; $\mathrm{MMT}$, manual muscle test, affected side; $\mathrm{HF}$, hip flexor; HE, hip extensor; KF, knee flexor; KE, knee extensor; MIT, maximal isokinetic voluntary contraction torque, affected side; BBS, Berg Balance Scale; 10MWT, 10-meter walking test; TUGT, Timed Up and Go Test; FAC, Functional Ambulation Category.

${ }^{*} P<0.05$ by Mann-Whitney U-test.

maximal isokinetic voluntary contraction torque, Berg Balance Scale, and Functional Ambulation Category. However, the WBV group showed greater improvement in the Berg Balance Scale, 10-m walk test, and Functional Ambulation Category. The results suggest that additional training with WBV may effectively improve the balance and gait performance of subacute stroke patients. We speculate that the improvement in balance through WBV therapy is closely related to improvements in the functional level and speed of walking.

Previous studies have reported that WBV therapy can improve balance through several mechanisms, including the modulation of the excitability of the spinal motoneuronal pool (Kipp et al., 2011), the activation of motor units (Pang et al., 2006), and improved proprioception (Fontana et al., 2005). The WBV platform generates vertical oscillations and horizontal movements. The contact surface of the platform transmits vibration stimuli from the feet to the rest of the body (Yang et al., 2015). WBV therapy is thought to activate the Ia and II afferent fibers (Nardone et al., 2001) and tonic vibration reflexes by detecting the stretch of the muscle (Fontana et al., 2005). The effects can improve postural control and thus enhance proprioceptive function. These neuromuscular responses have been associated with improvements in the muscular strength and power of the lower extremities, flexibility, gait speed, and balance, as well as reduced pain and risk of falls (Cochrane, 2011). Evidence also suggests that in young adults, WBV can exert a training effect on muscles by increasing tissue oxygenation (Games and Sefton, 2013), blood perfusion (Kerschan-Schindl et al., 2001), and intramuscular temperature (Cochrane et al., 2010). 
A previous study using the Barthel index suggested that WBV therapy is more effective in improving functional performance than conventional rehabilitation (Van Nes et al., 2006). Another study reported that WBV therapy resulted in significant improvements in the Barthel index in both a WBV group and a control group exercising with music (Merkert et al., 2011). Huh et al. (2015) found that balance control training improves both balance and gait. WBV stimulation appears to enhance weight-shifting to the hemiplegic side and improve balance control, thereby effectively improving gait performance.

Several studies have reported mobility improvements after WBV therapy. Merkert et al. (2011) reported better performance on the Timed Up and Go Test in a WBV group after 3 weeks of therapy. Likewise, Silva et al. (2014) found that WBV therapy improved performance in the Timed Up and Go Test and 6-min walk test. Chan et al. (2012) observed significant improvements in walking speed after WBV therapy. Choi et al. (2017) found that WBV combined with treadmill training improved the walking speed, step length, and stride length of chronic stroke patients. Priplata et al. (2003) reported that WBV is useful for stimulating proprioception and can result in long-lasting postural improvement.

In contrast, other studies have concluded that WBV therapy does not significantly improve the postural balance or body function of stroke patients. Lau et al. (2012) found that a leg exercise protocol using WBV therapy was no more effective in improving neuro-motor performance besides reducing the incidence of falls in chronic stroke patients. Other studies observed no significant postural improvements irrespective of the outcome measures used and regardless of whether the control groups underwent the same training without WBV (Lau et al., 2012; Martin et al., 2013) or with WBV (Brogardh et al., 2012). Van Nes et al. (2006) reported that although a 6-week WBV therapy improved balance, the improvement was not more significant than that in a control group.

A recent review concluded that the effects of WBV therapy on the muscle strength and mobility of stroke patients remain equivocal (Sañudo et al., 2018). We assume that this uncertainty is partly due to factors such as differences in patients' functional states and a lack of standardization in the frequency, duration, and intensity of the treatment. Despite this uncertainty, our results suggest that WBV therapy may effectively improve balance and gait performance in subacute stroke patients. Because it showed additional benefits to conventional physical therapy, we consider it a clinically useful tool. WBV therapy has no complications, such as dizziness, lower limb soreness and fatigue, or an itching sensation (Tankisheva et al., 2014).

Furthermore, WBV can provide continuous treatment. Just standing on the vibrator platform can keep the patient's muscle spindles active. It can thus be used as an alternative to conventional rehabilitation treatment, especially in the absence of a physical therapist, to improve the standing balance of subacute stroke patients.

This study has certain limitations. First, it was conducted with a small sample size and a short follow-up period. Thus, further research on a large population with a long follow-up period is required to determine the clinical efficacy of WBV therapy. Second, because we included only patients who were able to walk and stand, our results may not be generalizable to patients with different functional states. Despite its limitations, this study suggests that additional training with WBV may effectively improve the balance and gait performance in subacute stroke patients.

\section{CONFLICT OF INTEREST}

No potential conflict of interest relevant to this article was reported.

\section{ACKNOWLEDGMENTS}

This work was supported by the Dong-A University research fund.

\section{REFERENCES}

Brogardh C, Flansbjer UB, Lexell J. No specific effect of whole-body vibration training in chronic stroke: a double-blind randomized controlled study. Arch Phys Med Rehabil 2012;93:253-258.

Chan KS, Liu CW, Chen TW, Weng MC, Huang MH, Chen CH. Effects of a single session of whole body vibration on ankle plantarflexion spasticity and gait performance in patients with chronic stroke: a randomized controlled trial. Clin Rehabil 2012;26:1087-1095.

Choi W, Han D, Kim J, Lee S. Whole-body vibration combined with treadmill training improves walking performance in post-stroke patients: a randomized controlled trial. Med Sci Monit 2017;23:49184925 .

Cochrane DJ. The potential neural mechanisms of acute indirect vibration. J Sports Sci Med 2011:10;19-30.

Cochrane DJ, Stannard SR, Firth EC, Rittweger J. Comparing muscle temperature during static and dynamic squatting with and without 
whole-body vibration. Clin Physiol Funct Imaging 2010;30:223-229.

Fontana TL, Richardson CA, Stanton WR. The effect of weight bearing exercise with low frequency whole body vibration on lumbosacral proprioception: a pilot study on normal subjects. Aust J Physiother 2005;51:259-263.

Games KE, Sefton JM. Whole-body vibration influences lower extremity circulatory and neurological function. Scand J Med Sci Sports 2013;23: 516-523.

Huh JS, Lee YS, Kim CH, Min YS, Kang MG, Jung TD. Effects of balance control training on functional outcomes in subacute hemiparetic stroke patients. Ann Rehabil Med 2015;39:995-1001.

Kerschan-Schindl K, Grampp S, Henk C, Resch H, Preisinger E, Fialka-Moser V, Imhof $\mathrm{H}$. Whole-body vibration exercise leads to alterations in muscle blood volume. Clin Physiol 2001;21:377-382.

Kipp K, Johnson ST, Doeringer JR, Hoffman MA. Spinal reflex excitability and homosynaptic depression after a bout of whole body vibration. Muscle Nerve 2011;43:259-262.

Lau RWK, Yip SP, Pang MYC. Whole-body vibration has no effect on neuromotor function and falls in chronic stroke. Med Sci Sports Exerc 2012;44:1409-1418.

Martin PJ, Ferrero CM, Menendez H, Martin J, Herrero MA. Effects of whole-body vibration on muscle architecture, muscle strength, and balance in stroke patients: a randomized controlled trial. Am J Phys Med Rehabil 2013;92:881-888.

Martinez F, Rubio JA, Ramos DJ, Esteban P, Mendizabal S, Jimenez F. Effects of 6-week whole body vibration training on the reflex response of the ankle muscles: a randomized controlled trial. Int J Sports Phys Ther 2013;8:15-24.

Merkert J, Butz S, Nieczaj R, Steinhagen-Thiessen E, Eckardt R. Combined whole body vibration and balance training using Vibrosphere ${ }^{\circledR}$ : improvement of trunk stability, muscle tone, and postural control in stroke patients during early geriatric rehabilitation. Z Gerontol Geriat 2011;44:256-261.
Nardone A, Galante M, Lucas B and Schieppati M. Stancecontrol is not affected by paresis and reflex hyperexcitability: the case of spastic patients. J Neurol Neurosurg Psychiatry 2001;70:635-643.

Pang MY, Harris JE, Eng JJ. A community-based upper-extremity group exercise program improves motor function and performance of functional activities in chronic stroke: a randomized controlledtrial. Arch Phys Med Rehabil 2006;87:1-9.

Priplata AA, Niemi JB, Harry JD, Lipsitz LA, Collins JJ. Vibrating insoles and balance control in elderly people. Lancet 2003;362:1123-1124.

Sañudo B, Taiar R, Furness T, Bernardo-Filho M. Clinical approaches of whole-body vibration exercises in individuals with stroke: a narrative revision. Rehabil Res Pract 2018;2018:8180901.

Silva AT, Dias MPF, Calixto R Jr, Carone AL, Martinez BB, Silva AM, Honorato DC. Acute effects of whole-body vibration on the motor function of patients with stroke: a randomized clinical trial. Am J Phys Med Rehabil 2014;93:310-319.

Tankisheva E, Bogaerts A, Boonen S, Feys H, Verschueren SMP. Effects of intensive whole body vibration training on muscle strength and balancein adults with chronic stroke: a randomized controlledpilot study. Arch Phys Med Rehabil 2014;95:439-446.

Torvinen S, Kannu P, Sievänen H, Jarvinen TAH, Pasanen M, Kontulainen S, Jarvinen TLN, Jarvinen M, Oja P, Vuori I. Effect of a vibration exposure on muscular performance and body balance: randomized cross-over study. Clin Physiol Funct Imaging 2002;22:145-152.

Van Nes IJW, Latour H, Schils F, Meijer R, van Kuijk A, Geurts ACH. Long-term effects of 6-week whole-body vibration on balance recovery and activities of daily living in the postacute phase of stroke: a randomized, controlled trial. Stroke 2006;37:2331-2335.

Yang X, Wang Pu, Liu C, He C, Reinhardt JD. The effect of whole body vibration on balance, gait performance and mobility in people with stroke: a systematic review and meta-analysis. Clin Rehabil 2015;29: 627-638. 